\title{
Aesthetic and Musicological Interpretation of the Fifth Erhu Rhapsody by Wang Jianmin
}

\author{
Wang Juanjuan ${ }^{1}, \mathrm{Li} \mathrm{Jia}^{2, *}$ \\ ${ }^{1}$ College of Music, Qingdao University, Qingdao, Shandong, China \\ ${ }^{2}$ College of Music, Shanxi Normal University, Taiyuan, Shanxi, China \\ *Corresponding author
}

Keywords: Interpretation, the Fifth Erhu Rhapsody, Wang Jianmin

\begin{abstract}
On the road of erhu art pursuing aesthetic modernity, Mr. Wang Jianmin's series of five erhu rhapsody undoubtedly pushes erhu art to a new realm and a new height. Especially in "The Fifth Erhu Rhapsody," whether in terms of structure, mode, and musical language, or the challenge and innovation of performance techniques, Mr. Wang Jianmin not only moderately uses national music elements but strives to demonstrate a particular characteristic of national music. Also, strive to pursue the continuous innovation of erhu art, seek to combine tradition with modernity, nationality, and globality, and consciously pursue the aesthetic modernity of erhu art.
\end{abstract}

\section{Introduction}

Music performance aesthetics is an essential branch of music aesthetics, which reveals the essence and internal laws of music performance from the perspective of music performance practice. Music performance is a special kind of human activity, and its exceptional performance lies in its second degree of creativity. From the perspective of the three main links of music practice: music creation-music performance-music appreciation, music performance is in the middle of this chain, which connects music creation and appreciation. In other words, the musical performance artist connects the composer and the audience. In the sense of innovation, music performance is a creative act that is as important as music creation, and music performance itself is also a kind of creation.

\section{The aesthetic characteristics of the performance and interpretation of the Fifth Erhu} Rhapsody

As we all know, music is the art of time, realized through the performance of musical performance artists, and musical performance is an art form in which art production and art appreciation occur at the same time. In the process of music performance, different performers have different understanding and processing methods of the works, so a variety of performance genres and unique artistic expressions have been formed. If the performer wants to interpret the work better, he needs to have an in-depth understanding of the form and performance of the work itself and the composer. The author has studied piano since childhood, and systematically studied and 
performed erhu writers in different periods. Faced with the new work of "The Fifth Erhu Rhapsody---Anthem", the author hopes that Dao will pass through serious thinking and comprehension as well as in In the past performance experience, we have sought and explored the performance methods of the cooperative products, so that more works can better convey the composer's emotions and also bring more wonderful artistic feelings to the audience.

\section{Emotional Aesthetic Interpretation of Works}

"The Fifth Erhu Rhapsody-Hymn" is a work commissioned by the composer Wang Jianmin at the final stage of the 12th Golden Bell Awards Erhu Competition. On the occasion of such a heavyweight competition, it coincides with the 70th birthday of the People's Republic of China. Mr. Wang Jianmin specially added the first subtitle of the Erhu Rhapsody series to this "The Fifth Erhu Rhapsody"-"Anthem", in order to promote the spirit of the top professional awards in music, and express his praise for the motherland. A gift to the motherland. China is a country rich in cultural resources. Any artistic innovation cannot be separated from the nourishment of culture, and the innovation of Chinese music cannot be separated from the root of Chinese national culture. Mr. Wang Jianmin borrowed the erhu, a traditional Chinese national instrument, to create and improve folk music in the form of rhapsody, and brought a series of erhu rhapsody works that are full of traditional Chinese national elements but also give consideration to innovation and melody.

"The Fifth Erhu Rhapsody" is one of the strongest styles in the "Erhu Rhapsody" series. The "Adagio" and "Lyrical Paragraph" of the work occupy more space than the "Adagio" of the previous four Rhapsodies. In the whole song, the space is much larger, and the melody lines are more obvious. This shows the diversity of Wang Jianmin's creative styles in the same genre. The whole song is melodious and open, depicting the vast and magnificent scenery of the Mongolian people, and the enthusiastic and generous people, reflecting his deep friendship for the motherland. The composer extracts the rhythms of Mongolian folk songs such as "Hange" and "Chang Tune", imitating the most characteristic Mongolian art forms such as "Matouqin" and "Humai" to write. Mr. Wang Jianmin's creation does not simply use Chinese folk music elements, but extracts the core tones, expands them, and combines them with modern Western composition techniques, seeking a balance between tradition and modernity, and East and West. "The Fifth Erhu Rhapsody" combines Chinese folk music materials with modern Western composition techniques, striving for a Chinese style and Chinese style.

The rational analysis of the music score is the key link before the performance. The music score is the rational expression of the composer's emotions. How to rationally analyze the emotions hidden in the music score is presented through the instrument in the player's hands. It is a professional quality of the player. reflect. First of all, the analysis of the music score can start from the title of the work. From the title of "The Fifth Erhu Rhapsody-Hymn", it can be seen that this song is a rhapsody directly indicating the structural characteristics of its work. Next, it is necessary to deeply interpret the musical vocabulary of the chart, starting from the logical relationship of the musical structure, section, phrase, and festival of the work, and then to analyze the performance techniques such as mode tonality, intensity, and speed. It is necessary After careful consideration and reconsideration. Finally, grasp the unique sound of the erhu instrument to grasp the dynamics and expressiveness of the melody during performance.

\section{Aesthetic interpretation of second creation}

Music performance itself is "second creation." The most attractive aspect of music performance is its non-replicability. Every performance is unique. Each musical work is a presentation of the composer's expression of his emotions through creation. The work itself marks the composer's 
personal style characteristics and aesthetic experience, has the integrity of independent presentation, and is displayed in the musical work, that is, the spectrum. The presentation of an excellent work not only requires the author to create a musical work, but also requires the performer, through the accumulation of learning and experience, to finally present a work that satisfies the composer himself, the performer, and the audience. The processing is an important manifestation of the performer's personal style.

Music performance needs to provide the audience with a beautiful feeling, and the performer needs to constantly "run in" with the work. It is far from enough to perform sight reading only according to the melody of the chart. It is necessary to obtain a deep impression of the work through continuous practice, and then integrate personal emotions into the performance of the work, and finally present a song that can be presented to the audience. Contagious musical works of contemporary "environment". Liu Xie's "Wen Xin Diao Long.Shen Si" said that "the husband is thinking far away, Wantu competes in a vacant position, and is carved invisible. Mountain climbing is full of love in the mountains, while watching the sea is overflowing in the sea. How talented I am will be Wind and cloud drive together." How to make nihility emotions and intangible thoughts concretized and portrayed by music performance through imagination and feelings, as mentioned in this article, requires creators to have the ability to empathize and borrow emotions, and then sincerely convey these emotions to the audience through performance portrayal expressions.

\section{Innovative Performance Techniques-Discussing the Aesthetic Interpretation of Microphonic Techniques}

Skillful performance techniques are closely linked to the artistic connotation of the work. The perfect presentation of a musical work cannot be separated from the combination of "dao" and "technique". "The Fifth Erhu Rhapsody-Hymn" adopts the genre of "Rhapsody", the music is based on the music style of Inner Mongolia, and the "dazzling" performance technique is used to give full play to the performer's performance skills. As far as the genre of "Rhapsody" is concerned, its characteristic is that it must embody the "dazzling performance" of the music. In order to strengthen the test of the performer's technical ability, composer Wang Jianmin, the performance of the "dazzling technique" technique in the music is the focus of this work. , And in the current erhu works using microphonic sounds combined with traditional language, Mr. Wang Jianmin was the first to try. This pioneering move has also prompted a driving innovation in erhu playing techniques. For erhu players, what needs to be emphasized is how to learn a new technique to perfectly interpret the emotions that the music wants to express. Therefore, the author simply sorts out the theoretical aspects, and analyzes and explains more from the perspective of performance. The emergence of microtones will open up a precedent for melody innovation in the field of erhu creation, and will also have a profound impact on the growth of erhu performance techniques.

According to the chart record of "The Fifth Erhu Rhapsody-Hymn", Mr. Wang Jianmin explained its four rising and falling notes, and the details are shown in the following figure (see chart example 1)

Description of rising and falling tone marks 


\section{\$ 升高四分之一音 \\ \#升高四分之三音 \\ 降低四分之一音 \\ b 降低四分之三音}

\section{Spectrum Example 1}

It can be seen from the example of the score that the four differential tone notations used by Mr. Wang Jianmin are to raise one-quarter and three-quarter notes, and to lower one-quarter and three-quarter notes. The quarter tone is generally believed by music theorists to subdivide the twelve equal temperament. From a theoretical perspective, the interval can be subdivided into infinitely small, and this auditory narrower The use of microphonic sound is very necessary in the creation of erhu, which undoubtedly broadens the expressive power of erhu. Next, the author analyzes the differential music spectrum examples used in the work

In the first example, the introductory paragraphs 8-9 of the whole song. (See Example 2)

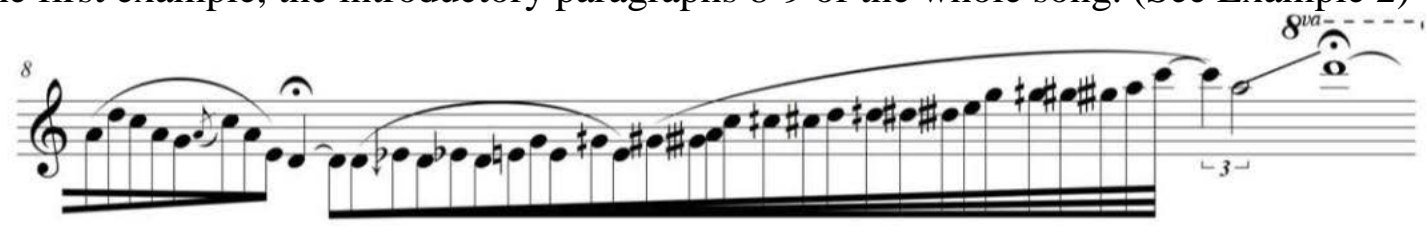

Spectrum example 2

The second example is the middle section of the Huacai section of the whole song. (See spectrum example 3)

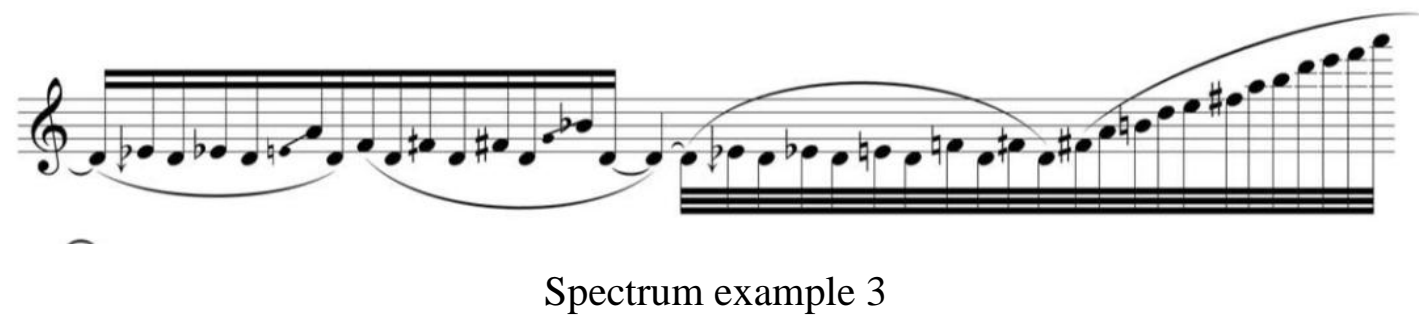

The third example is the allegro paragraph in the last paragraph of the whole district. (See spectrum example 4) 


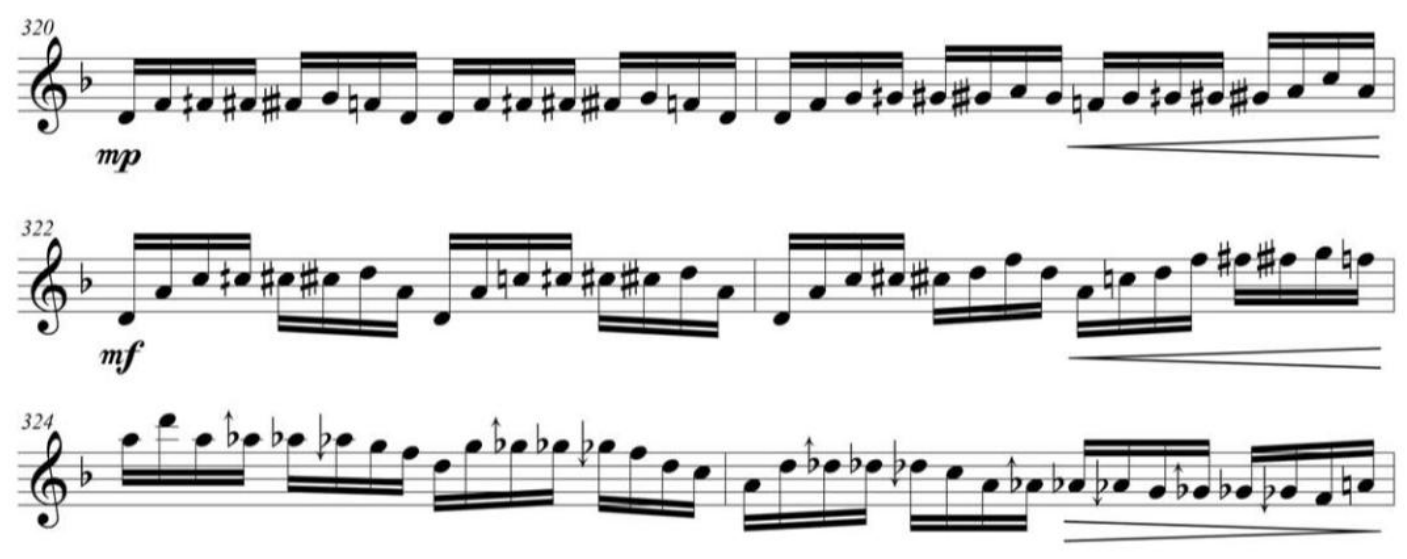

Spectrum example 4

It can be seen from the above-mentioned notation examples that the author arranges the microphonic performance in the key sections of the whole song. The first place (see Example 2) is at the 8-9th bar of the ending sentence of the intro paragraph of the whole song. The composer arranges the microtones according to the pitch and distributes them in the chromatic scale. After a dense arrangement of notes, the two octaves of the upward span are arranged to make the music more powerful and direct the theme. The second part (see Example 3) appears in the huacai paragraph of the whole work, which is similar to the first microphonic paragraph. Through the addition of the microphonic sound and alternately with the open string D sound, the elegant and delicate flavor of Inner Mongolian music is instantly added to lead to the following phrases. In the third place (see Example 4), the composer distributes the microtones in the climax of the whole song. Adding microtones to fast sixteenth note paragraphs will undoubtedly increase the difficulty of performance, making it more difficult to control the pitch and granularity. The fingering arrangement of the microtones is very important. The three examples of microphonic scores can clearly see how cleverly arranged fingerings are directly related to the difficulty of playing. When designing fingerings, the author is basically based on the erhu string method and timbre, tries to avoid the thinking of changing the same finger to set it, and must ensure the granularity and intonation of the notes. The same fingering may not be suitable for everyone, and it is necessary to proceed according to the unity of the tone of the finger and the chord.

\section{Conclusion}

As the "father of Rhapsody" composed in Chinese national instrumental music, Mr. Wang Jianmin has made significant contributions to enriching the repertoire of contemporary erhu works, and even the discipline construction and development of erhu performance, leading the development of China's erhu career. His erhu music creation corresponds to the public's aesthetics and unique personality. Strive to pursue continuous renewal and exploration in the design of erhu music, and finally, effectively combine the "Chinese flavor" with "the characteristics of the times." In particular, his five Erhu Rhapsody, whether in terms of creative techniques, musical structure, style characteristics, or performance techniques challenge and innovation, maintain the elements of national music and has superb technical methods. His creation uses modern composing techniques that are, based on preserving the recognizability of the original ecological folk songs. He has made great innovation and created a new peak in China's erhu playing career. 


\section{References}

[1] Qiao Jianzhong. (2000). A musical instrument and a century - - A century-old view of Erhu art. Music Research, 2000(01), 36-44.

[2] Qiao Jianzhong. (2017). Tradition, modernity and others-Deng Jiandong's piano sound. People's Music: Critic Edition, 2017 (08), 4-8.

[3] Qiao Jianzhong. (2011). On the modern spirit of erhu art. People's Music: Critic Edition, 2011 (05), 46-49, 95-96.

[4] Wang Jianmin. (2014). Composing new national rhymes and expressing Chinese feelings_-Review and reflection on the creation of "The First Erhu Rhapsody". People's Music, 2014 (03), 31-34.

[5] Wang Jianmin. (2003). Originated from folk root system tradition-"The Second Erhu Rhapsody" creation notes. People's Music, 2003(09), 2-5.

[6] Wang Jianmin. (2000). The study of melody. People's Music, 2000(2), 13-17.

[7] Wang Jianmin. (1999). On melody progression-a series of papers on melody. Journal of Nanjing University of the Arts (Music and Performance Edition), 1999(01), 3-12.

[8] Kong Zhixuan. (2020). A contemporary hymn from the grassland-Wang Jianmin's "The Fifth Erhu Rhapsody" Creation Comment. People's Music, 2020(6).

[9] Cheng Lin. (2019, November 25). Looking for "Crazy" Forward--A Comment on Wang Jianmin's Erhu Rhapsody. China Art News, No. 005, Hundred Schools of Art.

[10] Zhu Weiting. (2017). An analysis of the creative characteristics of Wang Jianmin's "The Second Erhu Rhapsody". Music creation, 2017(2), 129-131.

[11] Chen Chunyuan. (2017). Wang Jianmin's "Erhu Rhapsody Series" fingering application analysis and related thinking. People's Music, 2017(10), 38-41.

[12] Ru Yi. (2007). On the significance of Wang Jianmin's erhu works to the development of erhu art. (Doctoral dissertation, Shanghai Conservatory of Music).

[13] Ru Yi. (2008). From Wang Jianmin's Erhu Rhapsody to see his creative ideas and musical characteristics. Chinese Music, 2008(03), 219-221.

[14] Ru Yi. (2007). On the significance of Wang Jianmin's erhu works to the development of erhu art. Shanghai: Shanghai Conservatory of Music Press.

[15] Ru Yi. (2008). Wang Jianmin's Erhu Rhapsody Creation and Performance Analysis__Also on the innovative development of contemporary Erhu performance technology. Huang Zhong-Journal of Wuhan Conservatory of Music, 2008(04), 162-169.

[16] Zhang Yue. (2019, December 13). From "one madness" to "five madness", enhance the quality of erhu and show Chinese style. China Art News.

[17] Zhang Lei. Thoughts on the artistic characteristics and second creation of Wang Jianmin's "The Fourth Erhu Rhapsody". (Doctoral dissertation, China Conservatory of Music).

[18] Qian Renping, Tang Rong. (2013). Chinese breath, characteristics of the times-Wang Jianmin's "Erhu Rhapsody" creation. Chinese Music, 2013(003), 57-60.

[19] Qi Ping. (2005). Music performance art: theory and practice. Beijing: People's Music Publishing House. 